\title{
Gray Matter Volume Decrements in Preterm Children With Periventricular Leukomalacia
}

\author{
LEIRE ZUBIAURRE-ELORZA, SARA SORIA-PASTOR, CARME JUNQUE, DOLORS SEGARRA, NÚRIA BARGALLÓ, \\ NÚRIA MAYOLAS, CAMILA ROMANO-BERINDOAGUE, AND ALFONS MACAYA
}

\begin{abstract}
Department of Psychiatry and Clinical Psychobiology [L.Z.-E., S.S.-P., C.J., D.S.], Faculty of Medicine, University of Barcelona, Barcelona 08036, Spain; Radiology Department [N.B.], Centre de Diagnòstic per la Imatge, Clínic Hospital, Barcelona 08036, Spain; Institut de Diagnòstic per la Imatge [N.M.], Secció de Neurologia Infantil [C.R.-B., A.M.], Vall d'Hebron University Hospital,
\end{abstract} Barcelona 08035, Spain

\begin{abstract}
Periventricular leukomalacia (PVL) is the prototypic lesion in the encephalopathy of prematurity. Although PVL is identified by targeting cerebral white matter (WM), neuropathological and MRI studies document gray matter (GM) loss in cortical and subcortical structures. This study aimed to investigate the distribution of GM changes in children with a history of premature birth and PVL. Voxel-based morphometry was used to examine regional GM abnormalities in 22 children with a history of preterm birth and PVL. Preterms with PVL were compared with 22 terms and 14 preterms without PVL of similar GA and birth weight. GM and WM global volumetric volumes were found to decrease in comparison with both control groups. Regional GM volume abnormalities were also found: compared with their term peers, preterm children with PVL showed several regions of GM reduction. Moreover, PVL differed from preterms without PVL in the medial temporal lobe bilaterally, thalamus bilaterally, and caudate nuclei bilaterally. In addition, in our preterm sample with PVL, birth weight showed a statistical significant correlation with decreased GM regions. In conclusion, the voxel-based morphometry methodology revealed that PVL per se does involve GM reductions. (Pediatr Res 69: 554-560, 2011)
\end{abstract}

$\mathrm{P}$ eriventricular leukomalacia (PVL) is the most prevalent type of brain injury in the preterm infant. As such, it is associated with long-lasting cognitive, behavioral, and sensorymotor impairments. PVL comprises two different patterns of white matter (WM) injury, focal, and diffuse, and the most frequently affected regions are those located contiguous to the external angles of the lateral ventricles (1). The focal component implies necrosis of WM tissue and, depending on its size, is termed cystic (macroscopic) PVL or noncystic (microscopic) PVL (2). Diffuse PVL, usually presenting as hypomyelination and ventriculomegaly, is due to premyelinating oligodendrocyte loss and subsequent microgliosis and astrogliosis (3).

Although PVL characteristically affects cerebral WM (4), neuropathological studies in PVL samples have shown that

Received August 4, 2010; accepted January 4, 2011.

Correspondence: Carme Junqué, Ph.D., Department of Psychiatry and Clinical Psychobiology, Institut d'Investigacions Biomèdiques August Pi i Sunyer (IDIBAPS), University of Barcelona, C/Casanova, 143, CP: 08036 Barcelona, Spain; e-mail: cjunque@ub.edu

Supported by Grant SAF2005-007340 from the Ministerio de Ciencia y Tecnología and Grants 2009 SGR 00941 and 2009 SGR 0078 from the Generalitat de Catalunya. S.S.-P. and L.Z.-E. hold fellowships from the Ministerio de Educación y Ciencia (AP2005-0047 and AP2008-00935, respectively). gray matter (GM) lesions are frequently present in infants with PVL, indicating that WM injury is often accompanied by GM abnormalities in this preterm population $(5,6)$. MRI studies have reported global cortical GM and thalamic volume reductions in preterm neonates with PVL (7-9).

Voxel-based morphometry (VBM) is an automated procedure for quantifying GM and WM regional changes from a voxel-by-voxel analysis of MRI data (10). By using these procedures, we have previously found that premature adolescents without clinical WM lesions had WM reductions in several cortical and subcortical cerebral regions $(11,12)$ and that prematures at low risk of neurodevelopmental disorders showed GM reductions, mainly involving the temporal lobe (13). However, this technique has yet to be used in the study of preterm children with PVL.

The aims of this study were to investigate whether regional GM volume decreases were present in preterm children with PVL and, if so, to correlate these changes with clinical data. To exclude the effect of prematurity per se, we included a control group of preterms without PVL and similar GA and birth weight (BW).

\section{METHODS}

Subjects. The study was approved by the Ethics Committee of the University of Barcelona, and informed parental consent was obtained from all children's parents beforehand. Initially, between June 2008 and May 2009, we studied 36 children born preterm with PVL and belonging to a cohort being followed up at the Hospital Universitari Vall d'Hebron (Barcelona, Spain). All the participants underwent an assessment of their cognitive performance and an MRI study at the Hospital Clinic (Barcelona, Spain). They were all aged between 6 and 14 years at the time of the study. Inclusion criteria for this study were preterm birth defined as a GA $<37 \mathrm{wk}$ and signs of PVL demonstrated by neonatal ultrasound (US), plus PVL signs in the current-age MRI study. Exclusion criteria for the preterms with PVL were history of concomitant brain injuries such as grade III/IV peri-intraventricular hemorrhage, birth trauma, malformation, and infectious or metabolic encephalopathies. The preterm children with PVL were selected from a cohort of newborn patients diagnosed with PVL by neonatal US (WM periventricular echodensities present for more than $14 \mathrm{~d}$ or evolving into cysts) and born between 1994 and $2003(n=75)$. Causes of exclusion were GA $>37$ wk $(n=11)$, parents did not consent to the participation of their child in the study $(n=15)$, could not be contacted $(n=21)$, had received a full intelligence quotient

Abbreviations: BW, birth weight; CSF, cerebral spinal fluid; DARTEL, Diffeomorphic Anatomical Registration Through Exponentiated Lie algebra; GM, gray matter; PVL, periventricular leukomalacia; TIV, total intracranial volume; VBM, voxel-based morphometry; WM, white matter 
Table 1. MRI acquisition sequences

\begin{tabular}{cccc}
\hline MRI sequence & Full-term and non-PVL children & Preterm children with PVL & Purpose of MRI acquisition \\
\hline T1-weighted images & MPRAGE sequence in the sagittal & MPRAGE sequence in the sagittal orientation & VBM-DARTEL algorithm \\
& orientation $(\mathrm{TR} / \mathrm{TE}=2300 / 2.98 \mathrm{~ms} ;$ & $(\mathrm{TR} / \mathrm{TE}=2300 / 2.98 \mathrm{~ms}$; TI $=900 \mathrm{~ms} ;$ & analyses \\
& TI $=900 \mathrm{~ms} ; 256 \times 256$ matrix, flip & $256 \times 256$ matrix, flip angle $9^{\circ}, 1 \mathrm{~mm}^{3}$ & \\
& angle $9^{\circ}, 1 \mathrm{~mm}^{3}$ isotropic voxel) & isotropic voxel) & \\
& Axial orientation, TR/TE $=5533 / 88 \mathrm{~ms}$, & Axial orientation (TR/TE $=5150 / 99 \mathrm{~ms} ; 512$ & To identify any possible lesion \\
T2-weighted images & $122 \times 122 \mathrm{matrix}$, flip angle $90^{\circ}$, & $\times 307$ matrix, flip angle $120^{\circ}$, slice & not detectable on \\
& slice thickness $2 \mathrm{~mm}$, gap $=0.6 \mathrm{~mm}$ & thickness $5 \mathrm{~mm}$ with a 1.5 intersection gap) & T1-weighted images \\
\hline
\end{tabular}

MPRAGE, magnetic prepared rapid acquisition gradient echo; TR, time of repetition; TE, time of echo; TI, time of inversion.

Table 2. MRI classification of PVL

Grade 1 or mild PVL

White matter signal abnormality

a. Punctiform lesions of gliosis in the subcortical or paraventricular $\mathrm{WM}$ and/or small cystic lesions $(\leq 6)$

b. One focal area of gliosis in the PV-WM

c. Diffuse areas of gliosis in PV-WM

No reduction in WM volume

Normal size or mild enlargement of ventricles

No enlargement of subarachnoid space

Grade 2 or moderate PVL

White matter signal abnormality

a. Punctiform lesions of gliosis in the subcortical or paraventricular $\mathrm{WM}$ and/or small cystic lesions $(>6)$

b. Focal areas of gliosis in the PV-WM

c. Focal areas of gliosis in PV-WM with focal extension toward the subcortical or paraventricular WM (two areas)

d. Diffuse areas of gliosis in PV-WM with focal extension toward the subcortical or paraventricular WM (two areas)

Mild or moderate PV-WM reductions with focal subcortical or paraventricular reductions (two areas)

Moderate enlargement of ventricles. Focal irregularities in the ventricular walls (unilateral or bilateral)

No or mild enlargement of subarachnoid space

Grade 3 or severe PVL

White matter signal abnormality

a. Extensive confluent areas of PV gliosis with diffuse extension toward subcortical and paraventricular WM

b. Subcortical or paraventricular WM confluent areas of gliosis

c. Multicystic leukomalacia

Reductions in PV, paraventricular, and subcortical WM

Severe enlargement of ventricles. Diffuse irregularities in the ventricular walls (unilateral or bilateral)

Moderate or severe enlargement of subarachnoid space

Abbreviations: PV, periventricular; PVL, periventricular leukomalacia; $\mathrm{WM}$, white matter.

(FIQ) $<70(n=2)$, came for a first interview but declined the MRI $(n=3)$, or did not show WM impairment in the current MRI $(n=1)$. Neonatal and current clinical data were retrieved from a standardized database used in the prospective follow-up of the PVL cohort.

We used two different control groups, full-term children and preterms without PVL, from a cohort enrolled in previous MRI studies $(13,14)$. Both groups were recruited at the Pediatrics Service of the Hospital Clinic (Barcelona, Spain). Inclusion criteria for preterm children without PVL were GA and $\mathrm{BW}$ similar to the PVL group, a preterm birth with absence of major cerebral pathology (such as intraventricular hemorrhage, PVL, or WM injury detected by neonatal cranial US), and absence of neonatal morbidity (severe RDS, mechanical support, necrotizing enterocolitis, neonatal sepsis, or bronchopulmonary dysplasia). Any contraindication for MRI and a FIQ $\leq 70$ were grounds for exclusion in any participant. Full-term children were recruited among the friends or classmates of the preterm children without brain injury. The final sample comprised 22 preterm children with PVL, 14 preterms without PVL, and 22 full-terms, and their mean scores related to handedness, gender, and age at scan did not differ.

MRI acquisition and neuroradiological classification. MRI images were performed on a TIM TRIO 3T scanner (Siemens, Erlangen, Germany). A set

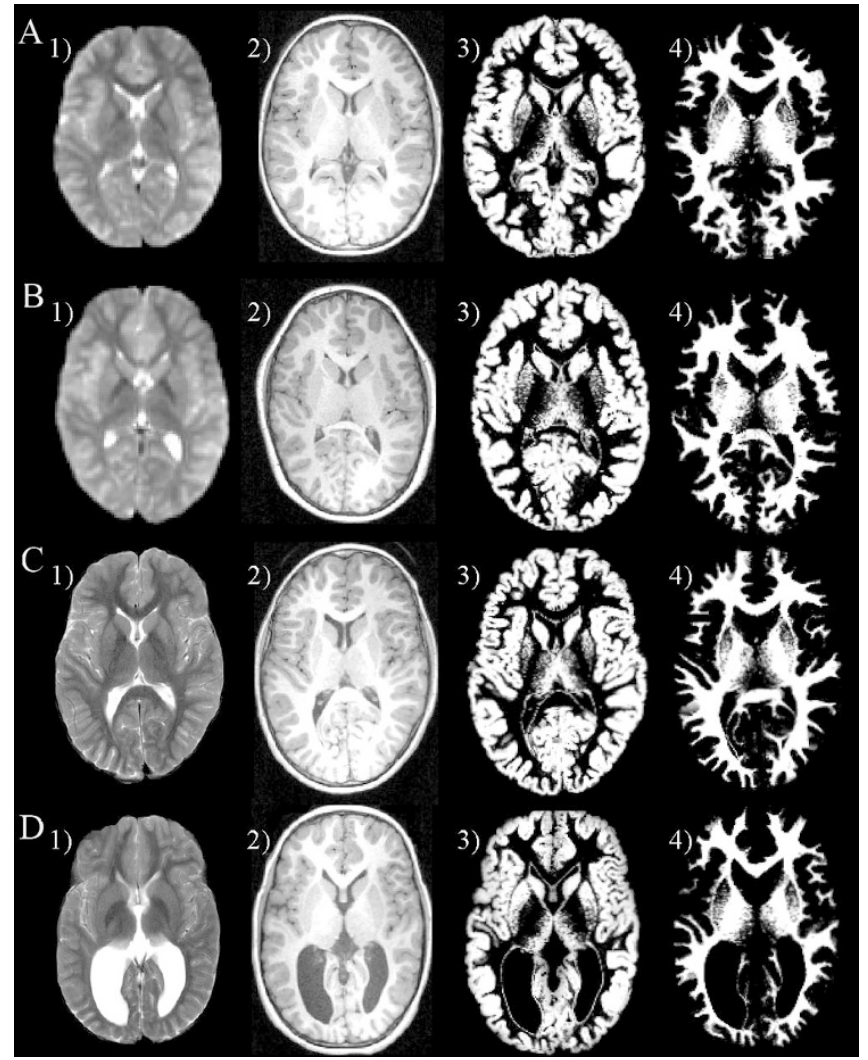

Figure 1. Control groups and range of severity of PVL. Rows: $(A)$ full-term; (B) non-PVL preterm; $(C)$ mild PVL; $(D)$ severe PVL. Columns: (1) T2weighted; (2) T1-weigheted; (3) GM segmentation; (4) WM segmentation.

of high-resolution three-dimensional T1-weighted and T2-weighted images were obtained (Table 1).

Two expert neuroradiologists evaluated all MRI scans to detect any brain abnormality in the control groups and to diagnose PVL in the current MRI. The severity of PVL was established in line with a set of criteria based on current MRI findings adapted from Flodmark et al. (15) (see Table 2). PVL patients were classified into three groups: mild, moderate, and severe. For the purpose of analysis, patients belonging to the moderate and severe PVL categories were grouped into a single category that we termed "severe PVL."

Image analysis. All the images were processed with automated SPM5 software (Statistical Parametric Mapping; The Wellcome Department of Imaging Neuroscience, Institute of Neurology, University College London, United Kingdom) running in MATLAB 7.0 (MathWorks, Natick, MA). The VBM method (10) was used to compare GM and WM volumes among the three groups (i.e. preterms with PVL, preterms without PVL, and full-term children) using the following steps: 1) the first, manual steps: anteroposterior commissure alignment and image reorienting of all images; 2) whole-brain segmentation of WM, GM, and cerebrospinal fluid (CSF) by means of tissue probability maps. The maps were generated from our own sample to improve the segmentation precision (Fig. 1). Total intracranial volume (TIV) was computed as the sum of these three cerebral tissues. 3) All the images to be analyzed were imported, and after an accurate registration of the images, we created our own template of the sample. All images were then warped to align 
Table 3. Neonatal and demographic characteristics of the samples

\begin{tabular}{|c|c|c|c|}
\hline & $\begin{array}{c}\text { Preterm with PVL, } \\
\text { mean } \pm \mathrm{SD}(n=22)\end{array}$ & $\begin{array}{l}\text { Preterm without PVL, } \\
\text { mean } \pm \mathrm{SD}(n=14)\end{array}$ & $\begin{array}{l}\text { Full-term, mean } \pm \mathrm{SD} \\
\quad(n=22)\end{array}$ \\
\hline \multicolumn{4}{|l|}{ Neonatal data } \\
\hline Gestational age (wk), median (range) & $29.5(26-36)$ & $32(30-34)$ & $40(38-41) \S \ddagger$ \\
\hline Birth weight $(\mathrm{g})$ & $1545.7 \pm 538.6$ & $1701.1 \pm 474.2$ & $3391.6 \pm 357.0 \S \ddagger$ \\
\hline Length $(\mathrm{cm})^{*}$ & $39.5 \pm 3.9$ & $42.4 \pm 4.5$ & $50.7 \pm 2.1 \S \ddagger$ \\
\hline Head circumference $(\mathrm{cm}) \dagger$ & $27.4 \pm 2.7$ & $29.8 \pm 2.6$ & $35.2 \pm 1.1 \S \ddagger$ \\
\hline \multicolumn{4}{|l|}{ Demographic data } \\
\hline Gender, M/F & $13 / 9$ & $10 / 4$ & $14 / 8$ \\
\hline Age at scan (y) & $8.7 \pm 1.8$ & $9.2 \pm 0.7$ & $9.3 \pm 0.6$ \\
\hline Right-handed, n (\%) & $17(77)$ & $12(86)$ & $22(100)$ \\
\hline
\end{tabular}

\footnotetext{
* Data available for 12 preterm with PVL, 14 preterm without PVL, and 22 full-term children.

$\dagger$ Data available for 12 preterm with PVL, 14 preterm without PVL, and 18 full-term children.

\$ Significant differences between full-term children in comparison with preterms with PVL and preterms without PVL.

$\S p \leq 0.001$.
}

them with the template using the SPM5 toolbox, the "Diffeomorphic Anatomical Registration Through Exponentiated Lie algebra" (DARTEL) algorithm (16). 4) Finally, all images were modulated, normalized to Montreal Neurological Institute (MNI) space, and smoothed with a full width at half-maximum of 8-mm Gaussian kernel.

Statistical analysis. Normality and homogeneity tests were first performed for all data. One-way ANOVA was used for normally distributed quantitative variables to compare the three groups of interest. The $\chi^{2}$ nonparametric test of independence for categorical variables was applied, as required. Post hoc analyses, when necessary, were applied to the three groups. Depending on homoscedasticity criteria, either a $t$ test with Bonferroni correction or Dunnett C test was used. All statistical analyses were computed using SPSS version 16.0 (SPSS, Inc., Chicago, IL).

For VBM analyses with the DARTEL algorithm, a family-wise error (FWE) correction was applied. Thus, corrected $p<0.05$ was determined as the threshold for statistical significance. Cluster threshold was set at 60 voxels. GM volume comparisons between groups were performed with statistical analyses of covariance. After whole-brain comparisons were performed, contrasts of interest ( $t$ test analyses) were the study group compared with preterms without PVL and with full-terms. For the contrast "preterm with PVL < preterm without PVL," a separate analysis was conducted to investigate any differences at the level of different regions of interest (ROI) in the basal ganglia (caudate, putamen, and globus pallidus) and thalamus, isolating the effect of PVL itself.

As a complement to the GM VBM-DARTEL analysis, WM regional volumes were also studied to investigate whether WM decreased regions in subjects with PVL were adjacent to GM reduced areas. Correlations of decremented regional GM volumes in the PVL preterm sample and clinical data (GA and BW) were performed through multiple regression analyses. Two covariates, age at scan and TIV, were introduced into each statistical design.

\section{RESULTS}

Clinical data. Neonatal and demographic characteristics of the samples are detailed in Table 3. As expected, full-term children differed significantly in neonatal data compared with both preterm groups. However, no significant differences in gender, laterality, or age at scan were found among the three groups. In accordance with the selection criteria of the preterm subgroups, prematures with PVL did not differ in GA and birth weight. The main clinical and neurological neonatal features of the PVL preterm group are detailed in Table 4.

Clinical MRI findings. One preterm children with a reported diagnosis of PVL in the neonatal US scan but with a current MRI study showing no signs of brain damage by visual inspection was eliminated from the VBM DARTEL study. Thus, 10 and 12 (seven were moderate and five severe) PVL preterm children were finally assigned to the mild and severe PVL groups, respectively. Visual inspection of the
Table 4. Clinical data of preterm PVL children

\begin{tabular}{|c|c|c|}
\hline & $\begin{array}{l}\text { PVL preterm } \\
\text { sample, n }(\%)\end{array}$ & $\begin{array}{c}\text { Non-PVL preterm } \\
\text { sample, } \mathrm{n}(\%)\end{array}$ \\
\hline \multicolumn{3}{|l|}{ Perinatal variables } \\
\hline Chorioamnionitis & $7 / 20(35)$ & \\
\hline Mild & $4 / 10$ & 2/14 (14) \\
\hline Severe & $3 / 10$ & \\
\hline Antenatal steroids & $16 / 20(80)$ & \\
\hline Mild & $9 / 10$ & $10 / 14(71)$ \\
\hline Severe & $7 / 10$ & \\
\hline Apgar score $<6$ at fifth min & 4/21 (19) & \\
\hline Mild & $3 / 10$ & $1 / 14(7)$ \\
\hline Severe & $1 / 11$ & \\
\hline Mechanical ventilation $>14 \mathrm{~d}$ & $4 / 21(19)$ & \\
\hline Mild & $2 / 10$ & $0 / 14(0)$ \\
\hline Severe & $2 / 11$ & \\
\hline Vaginal delivery & $9 / 22(41)$ & \\
\hline Mild & $2 / 10$ & $4 / 14(29)$ \\
\hline Severe & $7 / 12$ & \\
\hline Seizures & $3 / 22(14)$ & \\
\hline Mild & $1 / 10$ & $0 / 14(0)$ \\
\hline Severe & $2 / 12$ & \\
\hline IUGR & $1 / 22(5)$ & \\
\hline Mild & $0 / 10$ & $0 / 14(0)$ \\
\hline Severe & $1 / 12$ & \\
\hline SGA & $6 / 22(27)$ & \\
\hline Mild & $1 / 10$ & $3 / 14(21)$ \\
\hline Severe & $5 / 12$ & \\
\hline IVH grades I-II & $11 / 22(50)$ & \\
\hline Mild & $5 / 10$ & $0 / 14(0)$ \\
\hline Severe & $6 / 12$ & \\
\hline \multicolumn{3}{|l|}{ Neurological outcome } \\
\hline Mild dystonia & $3 / 22(14)$ & \\
\hline Mild & $0 / 10$ & $0 / 14(0)$ \\
\hline Severe & $3 / 12$ & \\
\hline Spastic diplegia & $3 / 22(14)$ & \\
\hline Mild & $2 / 10$ & $0 / 14(0)$ \\
\hline Severe & $1 / 12$ & \\
\hline Monoparesis & $2 / 22(9)$ & \\
\hline Mild & $0 / 10$ & $0 / 14(0)$ \\
\hline Severe & $2 / 12$ & \\
\hline Extra support at school & $4 / 22(18)$ & \\
\hline Mild & $1 / 10$ & $0 / 14(0)$ \\
\hline Severe & $3 / 12$ & \\
\hline
\end{tabular}

IUGR, intrauterine growth restriction; SGA, small for gestational age; IVH, intraventricular hemorrhage. 
Table 5. Brain volumetric data comparisons between preterms with and without PVL and full-terms

\begin{tabular}{|c|c|c|c|c|}
\hline Volumetric data $\left(\mathrm{cm}^{3}\right)$ & $\begin{array}{c}\text { Preterm with PVL, } \\
\text { mean } \pm \mathrm{SD}(n=22)\end{array}$ & $\begin{array}{l}\text { Preterm without PVL, } \\
\text { mean } \pm \mathrm{SD}(n=14)\end{array}$ & $\begin{array}{c}\text { Full-term, mean } \pm \mathrm{SD} \\
(n=22)\end{array}$ & Statistics $(p)$ \\
\hline Gray matter & $714.59 \pm 71.32$ & $795.68 \pm 86.63$ & $819.29 \pm 76.24$ & $F=10.89(\leq 0.001)^{* \dagger}$ \\
\hline White matter & $382.58 \pm 50.01$ & $426.76 \pm 46.21$ & $436.64 \pm 44.18$ & $F=7.99(\leq 0.001)^{* \dagger}$ \\
\hline Cerebrospinal fluid & $538.62 \pm 173.73$ & $516.85 \pm 172.56$ & $482.88 \pm 163.36$ & $F=0.60(0.552)$ \\
\hline Total intracranial volume & $1635.79 \pm 216.90$ & $1739.29 \pm 285.38$ & $1738.82 \pm 255.63$ & $F=1.17(0.318)$ \\
\hline
\end{tabular}

$* p \leq 0.001$.

$\dagger$ Significant differences between preterm with PVL group in comparison with both control groups (full-term and preterm without PVL children).

MRI studies of the full-term and preterm without PVL control groups did not reveal any brain abnormality.

VBM-DARTEL results. Global brain volume measurements are presented in Table 5. There were no differences among the three groups in the TIV or CSF volumes. However, the PVL preterm group showed statistically significant reductions in global GM and WM volumes compared with the preterm group without PVL and full-term children.

GM results. For GM whole brain comparisons, the main effect of group was statistically significant, $F=12.59$ ( $p<$ 0.05). Compared with full-term children, the preterm group with PVL had GM reductions in several regions: the left parietal and right frontal lobes, as well as bilaterally in the temporal lobes, including the medial temporal lobe (see in Fig. 2 and Table 6).

Compared with preterms without PVL, preterms with PVL had GM volume reductions in the medial temporal lobe bilaterally (see Fig. 2 and Table 6). Regarding the severity of the pathology, preterms with severe PVL showed lower GM values in the right thalamus $([-5-92], t=5.69, p=0.002)$ and in both medial temporal lobes $\left(\left[\begin{array}{lll}-23 & 0 & -21\end{array}\right], t=6.31\right.$, $p \leq 0.001 ;[24-2-21], t=5.40, p=0.002)$. However, preterms with mild PVL impairment and preterm children without PVL did not differ in any GM region.

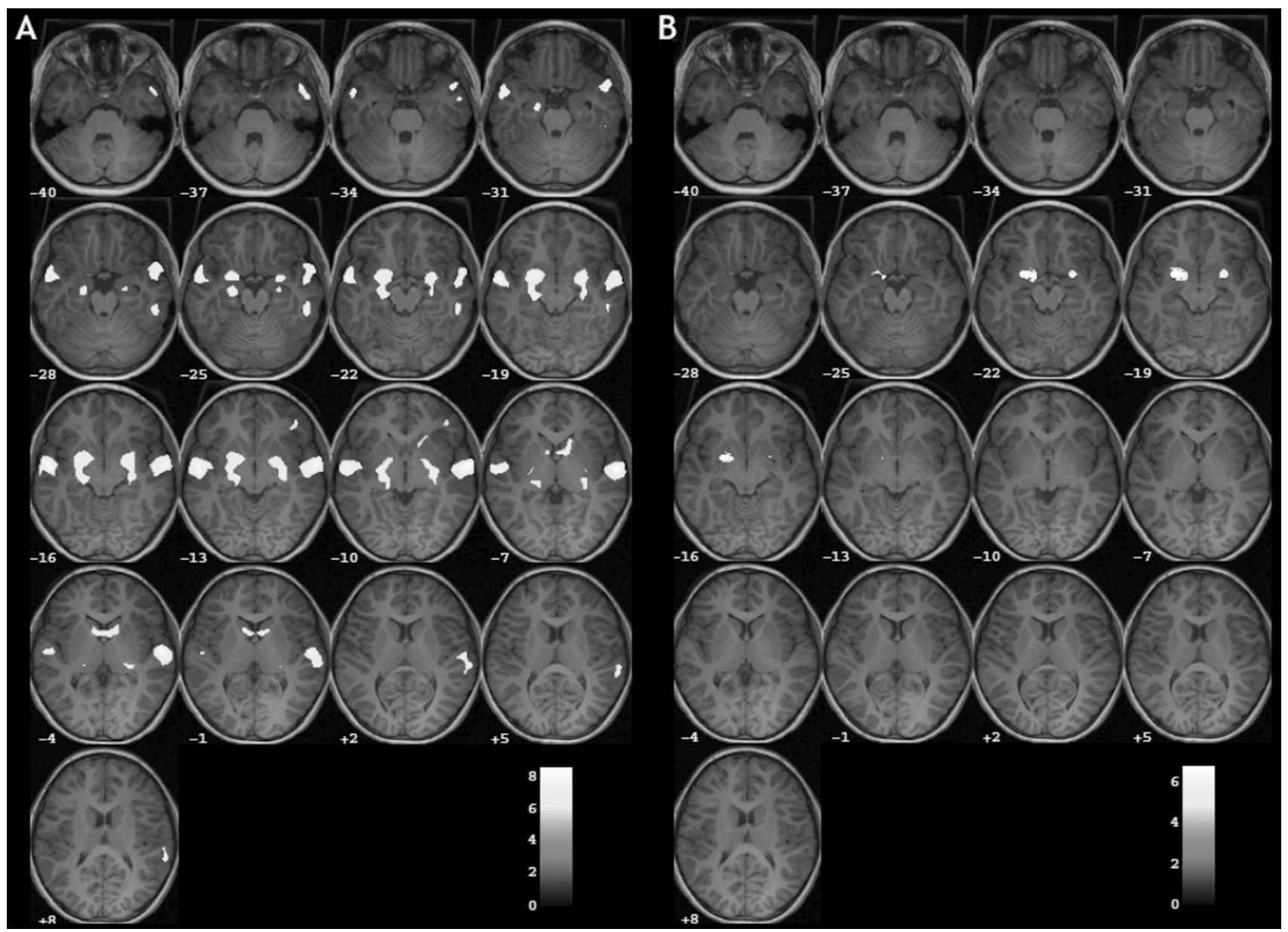

Figure 2. Structural MRI showing GM differences, presented according to radiological convention. Statistical parametric maps, where axial views showed decrements in the preterm group with PVL $(n=22)$ compared with $(A)$ their term $(n=22)$ and $(B)$ their preterm peers $(n=14)$. All the results are displayed on a term standard brain and are FWE-corrected at the $p<0.05$ level. 
Table 6. Regional brain GM decrements in preterm children with PVL compared with preterms without PVL and full-term children

\begin{tabular}{|c|c|c|c|c|c|c|}
\hline \multirow[b]{2}{*}{ Anatomical region (BA) } & \multirow{2}{*}{$\begin{array}{l}\text { Cluster size } \\
\left(\mathrm{mm}^{3}\right)\end{array}$} & \multirow{2}{*}{$\begin{array}{l}\text { Voxel-level } \\
(p \text { corrected })\end{array}$} & \multicolumn{3}{|c|}{ MNI coordinates } & \multirow[b]{2}{*}{$t$ statistic } \\
\hline & & & $x$ & $y$ & $z$ & \\
\hline \multicolumn{7}{|l|}{$\begin{array}{c}\text { Preterms with PVL }(n=22) v s \\
\text { full-terms }(n=22)\end{array}$} \\
\hline \multicolumn{7}{|l|}{ Parietal lobe } \\
\hline Postcentral gyrus (4) L & 537 & $<0.0001^{*}$ & -59 & -23 & 44 & 6.38 \\
\hline Inferior parietal lobule (40) L & 223 & $0.003 \dagger$ & -41 & -38 & 42 & 6.13 \\
\hline \multicolumn{7}{|l|}{ Frontal lobe } \\
\hline Middle frontal gyrus (11) R & 344 & $<0.0001^{*}$ & 42 & 35 & -12 & 6.18 \\
\hline \multicolumn{7}{|l|}{ Temporal lobe } \\
\hline Middle temporal gyrus (21) L & 3868 & $<0.0001^{*}$ & -60 & -14 & -12 & 7.95 \\
\hline Middle temporal gyrus (21) R & 7573 & $<0.0001^{*}$ & 62 & -14 & -11 & 8.14 \\
\hline Inferior temporal gyrus (20) R & 688 & $<0.0001 *$ & 53 & -41 & -27 & 6.99 \\
\hline Medial temporal lobe L & 8680 & $<0.0001 *$ & -26 & -5 & -20 & 7.28 \\
\hline Medial temporal lobe $\mathrm{R}$ & 7104 & $<0.0001 *$ & 24 & -6 & -18 & 7.17 \\
\hline \multicolumn{7}{|l|}{$\begin{array}{l}\text { Preterms with PVL }(n=22) \text { vs } \\
\text { preterms without PVL }(n=14)\end{array}$} \\
\hline Medial temporal lobe L & 2460 & $\leq 0.0001^{*}$ & -26 & -2 & -21 & 6.72 \\
\hline Medial temporal lobe $\mathrm{R}$ & 999 & $\leq 0.0001^{*}$ & 26 & -3 & -20 & 5.98 \\
\hline
\end{tabular}

The localization (MNI coordinates) corresponds to one representative voxel of the significant cluster. These coordinates show that $x$ increases from left ( - ) to right $(+) ; y$ increases from posterior $(-)$ to anterior $(+)$; and $z$ increases from inferior $(-)$ to superior $(+)$.

$* p \leq 0.001$.

$\dagger p<0.05$.

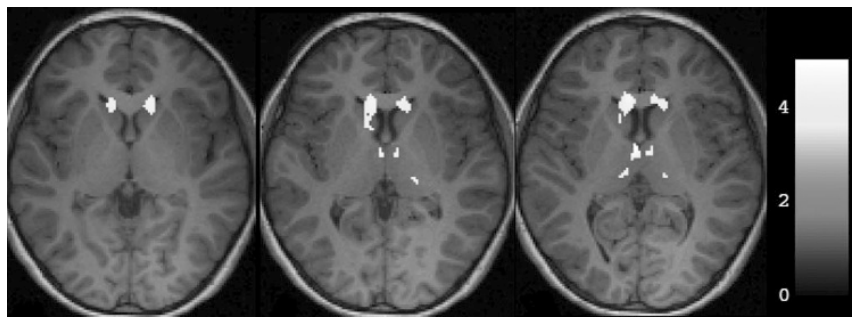

Figure 3. Basal ganglia and thalamus region of interest analysis, where decremented areas $(p<0.05)$ in preterms with PVL $(n=22)$ in comparison with preterms without brain injury $(n=14)$ are represented in yellow.

The ROI analyses showed that compared with preterms without PVL, preterms with PVL had GM reductions in the thalamus $([-5-92], t=5.00, p \leq 0.001 ;[15-265], t=$ $4.45, p=0.006)$ and in the head of the caudate nucleus $([-6$ $213], t=4.88, p \leq 0.001$; [12 6 20], $t=4.78, p \leq 0.001$ ) (see Fig. 3).

WM results. The effect of group regarding WM whole brain comparisons was also statistically significant, $F=13.35(p<$ 0.05). Compared with their term counterparts, preterms with PVL showed decreased volumes localized in the corpus cal$\operatorname{losum}([-3-141], t=7.61, p \leq 0.001)$, around the posterior horns of the lateral ventricle ( $[-27-35-3], t=6.26, p \leq$ 0.001 ; [30 - 35 -3], $t=6.16, p \leq 0.001)$, and bilaterally at the level of the centrum semiovale $([-29-2741], t=5.64$, $p \leq 0.001$; [29 - 57 35], $t=5.69, p \leq 0.001$; [20 - 24 41], $t=5.83, p \leq 0.001)$. Children with PVL differed from preterms without PVL in the pons ([11 -21 -29], $t=5.80$, $p \leq 0.001)$.

GM correlations with clinical variables. Significant correlations were observed between different GM areas and BW in the sample of preterm children with PVL. These regions were middle ([54 - 26 -14], $t=7.46, p \leq 0.001)$ and superior ([-59-14-6], $t=6.47, p \leq 0.001)$ temporal gyri and medial temporal lobe ([27 - 38 -12], $t=6.92, p \leq 0.001$ ). Correlation analyses between GM regional volumes and GA did not reach statistical significance at the corrected level. However, when the statistical maps were displayed uncorrected for multiple comparisons, the same temporal areas described for BW were observed as being relevant.

\section{DISCUSSION}

To our knowledge, this is the first MRI study in a sample of PVL preterms at school age to have investigated GM regional abnormalities by using an automated approach of MRI analysis. Compared with their term counterparts, preterm children with PVL showed GM reductions in the left parietal and right frontal and temporal lobes bilaterally, including medial temporal regions. Moreover, these medial temporal regions reached statistical significance when PVL children were compared with those preterms without brain injury. ROI analyses also showed differences in this contrast (preterm with PVL $<$ preterm without PVL) at the caudate nucleus and thalamus bilaterally. Thus, the GM decrements ultimately ascribable to PVL involve medial temporal lobe, thalamus, and caudate bilaterally.

Although cerebral WM is the most commonly affected tissue related to preterm labor (3), GM abnormalities are recognized to occur in the preterm population. Despite the fact that cortical and subcortical decremented GM regions have previously been reported in preterm samples at school age $(13,17-19)$, fewer reports have isolated samples of preterms with PVL to investigate cerebral GM at this time of life. The first study providing evidence of GM damage in association with PVL was carried out by Inder et al. (7) in a group of 10 neonates with PVL. Similar to our findings, they found that, using image-processing algorithms, decrements in total myelinated WM are accompanied by global cortical GM reductions in subjects with PVL compared with preterms without 
PVL and to full-term controls. Technical constraints of MRI analysis in the previous decade precluded a regional automatic quantification. In our study, the developmental age at which our sample was analyzed, coupled with the use of modern morphometric techniques such as VBM-DARTEL analysis, allowed the identification of discrete cerebral regions prone to suffer GM loss in a PVL context.

When we isolated the effect of PVL per se, we found decrements in subcortical structures such as medial temporal lobe, caudate, and thalamus. In the study by Peterson et al. (17) of 25 children born preterm, the cerebral structure with the highest and most significant percentage of injury was also a medial temporal structure. Interestingly, except for the medial temporal lobe, the pattern of subcortical GM reductions that we observed in vivo concurs with that described in a PVL postmortem study (5), where neuronal loss in the thalamus and basal ganglia was detected in a group of preterm infants featuring WM gliosis and focal periventricular necrosis. In fact, the thalamus is recognized as a region that is highly vulnerable to damage in preterm samples with PVL $(8,9,20,21)$, and caudate impairment has been frequently reported in preterm samples $(18,22)$. It appears that the decreased thalamic region is, with high probability, the dorsomedial nucleus. Impact to this region has been related to the cognitive outcome in premature samples with WM injury $(21,23)$. Marin-Padilla (24) reported in a neuropathologic study that the most impaired regions were those localized adjacent to perinatally acquired lesions, compared with the farthest ones, and that postinjury reorganization could also be affecting neurological and cognitive performance. Neonatal periventricular WM injury in preterm samples might lead to damage of subplate neurons, crucial in thalamocortical and corticocortical connections (25), and this could result in cortical and subcortical GM reductions. These GM reductions present in preterm samples with WM injury might be also related with the high incidence of seizures reported in preterms with brain injury $(26,27)$ as is the case for the abnormal cortical reorganization of some GM nuclei after cystic WM injury (28). Several GM regions in which preterms with PVL showed volume decrements were only correlated with the BW neonatal variable. These findings are in agreement with Cole et al. (29) who proposed that BW is a key marker of prematurity and Taylor et al. (30) who found that BW is the best predictor for long-term outcome. In our cohort, the higher predictive value of BW may be related to a higher incidence of small for GA infants in the severe PVL group. In addition, and as expected, the contrast analysis in which PVL itself was analyzed (compared with preterm children without PVL) showed that the greater the degree of brain injury, the larger the GM volume decrements. It is true that grades I-II intraventricular hemorrhage was clearly more frequent in the PVL than in the non-PVL preterm group, but it is accepted that, in the absence of ventricular dilation, mild intraventricular hemorrhage does not seem to involve additional significant risk of neurological sequelae for any given GA $(31,32)$.

Volumetric MRI studies in preterm samples with PVL have also reported WM abnormalities $(7,33)$. Corpus callosum decrements have frequently been reported in very preterm subjects without PVL at different ages (34-37), including with the use of VBM methodology $(11,38)$. In agreement with Davatzikos et al. (39), we found decrements in the corpus callosum in the group of PVL children group.

This study found several decremented cerebral areas in a sample of PVL preterms at school age by using an automatic approach. However, automatic measurements of cerebral changes from MRI studies in pediatric samples might have several limitations in the segmentation (40), registration (41), and/or normalization step (42), and thus our results might be taken into account with caution. Staudt et al. (43) advised against using GM and CSF volume values in PVL samples, because gliotic scars could be assigned to either GM or CSF. However, recent studies have recommended VBM-DARTEL as an alternative to standard approaches $(44,45)$. Specifically, VBM-DARTEL algorithm analyses improve intersubject registration (16), which is indispensable in preterm samples (13). This is especially the case for preterms with brain injury, where large ventricles are a common finding that hampers tissue class image alignment (46). Conversely, the relatively small sample size in our study resulted in a limited statistical power and the need for cautious interpretation of the present data.

Current understanding of PVL encompasses a wide spectrum of brain pathologies, which are reflected in the considerable variation in neurobehavioral outcomes of preterm babies in whom WM damage is readily identified in the neonatal period. Further studies including larger samples with different degrees of PVL are needed to investigate the patterns of WM and GM involvement in PVL subgroups and to identify their predictive values in terms of the development of specific cognitive abilities.

Acknowledgments. We thank C. Falco for technical assistance and S. Juanes for statistical support. We also thank the children and the families who participated in this study.

\section{REFERENCES}

1. Volpe JJ 2008 Neurology of the Newborn. WB Saunders, Philadelphia, PA, pp 359-379

2. Volpe JJ 2009 Brain injury in premature infants: a complex amalgam of destructive and developmental disturbances. Lancet Neurol 8:110-124

3. Volpe JJ 2003 Cerebral white matter injury of the premature infant-more common than you think. Pediatrics 112:176-180

4. Khwaja O, Volpe JJ 2008 Pathogenesis of cerebral white matter injury of prematurity. Arch Dis Child Fetal Neonatal Ed 93:F153-F161

5. Pierson CR, Folkerth RD, Billiards SS, Trachtenberg FL, Drinkwater ME, Volpe JJ, Kinney HC 2007 Gray matter injury associated with periventricular leukomalacia in the premature infant. Acta Neuropathol 114:619-631

6. Haynes RL, Folkerth RD, Trachtenberg FL, Volpe JJ, Kinney HC 2009 Nitrosative stress and inducible nitric oxide synthase expression in periventricular leukomalacia. Acta Neuropathol 118:391-399

7. Inder TE, Huppi PS, Warfield S, Kikinis R, Zientara GP, Barnes PD, Jolesz F, Volpe JJ 1999 Periventricular white matter injury in the premature infant is followed by reduced cerebral cortical gray matter volume at term. Ann Neurol 46:755-760

8. Lin Y, Okumura A, Hayakawa F, Kato K, Kuno T, Watanabe K 2001 Quantitative evaluation of thalami and basal ganglia in infants with periventricular leukomalacia. Dev Med Child Neurol 43:481-485

9. Ricci D, Anker S, Cowan F, Pane M, Gallini F, Luciano R, Donvito V, Baranello G, Cesarini L, Bianco F, Rutherford M, Romagnoli C, Atkinson J, Braddick O, Guzzetta F, Mercuri E 2006 Thalamic atrophy in infants with PVL and cerebral visual impairment. Early Hum Dev 82:591-595

10. Ashburner J, Friston KJ 2000 Voxel-based morphometry-the methods. Neuroimage 11:805-821

11. Giménez M, Junque C, Narberhaus A, Bargallo N, Botet F, Mercader JM 2006 White matter volume and concentration reductions in adolescents with history of very preterm birth: a voxel-based morphometry study. Neuroimage 32:1485-1498 
12. Soria-Pastor S, Gimenez M, Narberhaus A, Falcon C, Botet F, Bargallo N, Mercader JM, Junque C 2008 Patterns of cerebral white matter damage and cognitive impairment in adolescents born very preterm. Int J Dev Neurosci 26:647-654

13. Soria-Pastor S, Padilla N, Zubiaurre-Elorza L, Ibarretxe-Bilbao N, Botet F, CostasMoragas C, Falcon C, Bargallo N, Mercader JM, Junque C 2009 Decreased regional brain volume and cognitive impairment in preterm children at low risk. Pediatrics 124:e1161-e1170

14. Zubiaurre-Elorza L, Soria-Pastor S, Junque C, Vendrell P, Padilla N, Rametti G, Bargallo N, Botet F 2009 Magnetic resonance imaging study of cerebral sulci in low-risk preterm children. Int J Dev Neurosci 27:559-565

15. Flodmark O, Roland EH, Hill A, Whitfield MF 1987 Periventricular leukomalacia: radiologic diagnosis. Radiology 162:119-124

16. Ashburner J 2007 A fast diffeomorphic image registration algorithm. Neuroimage 38:95-113

17. Peterson BS, Vohr B, Staib LH, Cannistraci CJ, Dolberg A, Schneider KC, Katz KH, Westerveld M, Sparrow S, Anderson AW, Duncan CC, Makuch RW, Gore JC, Ment LR 2000 Regional brain volume abnormalities and long-term cognitive outcome in preterm infants. JAMA 284:1939-1947

18. Abernethy LJ, Cooke RW, Foulder-Hughes L 2004 Caudate and hippocampal volumes, intelligence, and motor impairment in 7-year-old children who were born preterm. Pediatr Res 55:884-893

19. Kesler SR, Reiss AL, Vohr B, Watson C, Schneider KC, Katz KH, MallerKesselman J, Silbereis J, Constable RT, Makuch RW, Ment LR 2008 Brain volume reductions within multiple cognitive systems in male preterm children at age twelve. J Pediatr 152:513-520

20. Srinivasan L, Dutta R, Counsell SJ, Allsop JM, Boardman JP, Rutherford MA Edwards AD 2007 Quantification of deep gray matter in preterm infants at termequivalent age using manual volumetry of 3-tesla magnetic resonance images. Pediatrics 119:759-765

21. Ligam P, Haynes RL, Folkerth RD, Liu L, Yang M, Volpe JJ, Kinney HC 2009 Thalamic damage in periventricular leukomalacia: novel pathologic observations relevant to cognitive deficits in survivors of prematurity. Pediatr Res 65:524-529

22. Abernethy LJ, Palaniappan M, Cooke RW 2002 Quantitative magnetic resonance imaging of the brain in survivors of very low birth weight. Arch Dis Child $87: 279-283$

23. Boardman JP, Craven C, Valappil S, Counsell SJ, Dyet LE, Rueckert D, Aljabar P, Rutherford MA, Chew AT, Allsop JM, Cowan F, Edwards AD 2010 A common neonatal image phenotype predicts adverse neurodevelopmental outcome in children born preterm. Neuroimage 52:409-414

24. Marín-Padilla M 1999 Developmental neuropathology and impact of perinatal brain damage. III: gray matter lesions of the neocortex. J Neuropathol Exp Neurol 58:407-429

25. Kostovic I, Judas M 2006 Prolonged coexistence of transient and permanent circuitry elements in the developing cerebral cortex of fetuses and preterm infants. Dev Med Child Neurol 48:388-393

26. Shah DK, Zempel J, Barton T, Lukas K, Inder T 2010 Electrographic seizures in preterm infants during the first week of life are associated with cerebral injury. Pediatr Res 67:102-106

27. Davis AS, Hintz SR, Van Meurs KP, Li L, Das A, Stoll B, Walsh MC, Pappas A, Bell EF, Laptook AR, Higgins RD 2010 Seizures in extremely low birth weight infants are associated with adverse outcome. J Pediatr 157:720-725

28. Marín-Padilla M 1997 Developmental neuropathology and impact of perinatal brain damage. II: white matter lesions of the neocortex. J Neuropathol Exp Neurol $56: 219-235$
29. Cole C, Binney G, Casey P, Fiascone J, Hagadorn J, Kim C, Lau J, Wang C, DeVine D, Miller K 2002 Criteria for determining disability in infants and children: low birth weight. Evid Rep Technol Assess (Summ) 70:1-7

30. Taylor HG, Minich N, Bangert B, Filipek PA, Hack M 2004 Long-term neuropsychological outcomes of very low birth weight: associations with early risks for periventricular brain insults. J Int Neuropsychol Soc 10:987-1004

31. Dyet LE, Kennea N, Counsell SJ, Maalouf EF, Ajayi-Obe M, Duggan PJ, Harrison M, Allsop JM, Hajnal J, Herlihy AH, Edwards B, Laroche S, Cowan FM, Rutherford MA, Edwards AD 2006 Natural history of brain lesions in extremely preterm infants studied with serial magnetic resonance imaging from birth and neurodevelopmental assessment. Pediatrics 118:536-548

32. Ancel PY, Livinec F, Larroque B, Marret S, Arnaud C, Pierrat V, Dehan M, N'Guyen S, Escande B, Burguet A, Thiriez G, Picaud JC, André M, Bréart G, Kaminski M 2006 EPIPAGE Study Group Cerebral palsy among very preterm children in relation to gestational age and neonatal ultrasound abnormalities: the EPIPAGE cohort study. Pediatrics 117:828-835

33. Tzarouchi LC, Astrakas LG, Zikou A, Xydis V, Kosta P, Andronikou S, Argyropoulou MI 2009 Periventricular leukomalacia in preterm children: assessment of grey and white matter and cerebrospinal fluid changes by MRI. Pediatr Radiol 39:1327-1332

34. Nosarti C, Rushe TM, Woodruff PW, Stewart AL, Rifkin L, Murray RM 2004 Corpus callosum size and very preterm birth: relationship to neuropsychological outcome. Brain 127:2080-2089

35. Isaacs EB, Edmonds CJ, Chong WK, Lucas A, Morley R, Gadian DG 2004 Brain morphometry and IQ measurements in preterm children. Brain 127:2595-2607

36. Rademaker KJ, Lam JN, Van Haastert IC, Uiterwaal CS, Lieftink AF, Groenendaal F, Grobbee DE, de Vries LS 2004 Larger corpus callosum size with better motor performance in prematurely born children. Semin Perinatol 28:279-287

37. Caldú X, Narberhaus A, Junque C, Gimenez M, Vendrell P, Bargallo N, Segarra D, Botet F 2006 Corpus callosum size and neuropsychologic impairment in adolescents who were born preterm. J Child Neurol 21:406-410

38. Nosarti C, Giouroukou E, Healy E, Rifkin L, Walshe M, Reichenberg A, Chitnis X, Williams SC, Murray RM 2008 Grey and white matter distribution in very preterm adolescents mediates neurodevelopmental outcome. Brain 131:205-217

39. Davatzikos C, Barzi A, Lawrie T, Hoon AH Jr, Melhem ER 2003 Correlation of corpus callosal morphometry with cognitive and motor function in periventricular leukomalacia. Neuropediatrics 34:247-252

40. Murgasova M, Dyet L, Edwards D, Rutherford M, Hajnal J, Rueckert D 2007 Segmentation of brain MRI in young children. Acad Radiol 14:1350-1366

41. Crinion J, Ashburner J, Leff A, Brett M, Price C, Friston K 2007 Spatial normalization of lesioned brains: performance evaluation and impact on fMRI analyses. Neuroimage 37:866-875

42. Wilke M, Holland SK, Myseros JS, Schmithorst VJ, Ball WS Jr 2003 Functional magnetic resonance imaging in pediatrics. Neuropediatrics 34:225-233

43. Staudt M, Pavlova M, Bohm S, Grodd W, Krageloh-Mann I 2003 Pyramidal tract damage correlates with motor dysfunction in bilateral periventricular leukomalacia (PVL). Neuropediatrics 34:182-188

44. Yassa MA, Stark CE 2009 A quantitative evaluation of cross-participant registration techniques for MRI studies of the medial temporal lobe. Neuroimage 44:319-327

45. Bergouignan L, Chupin M, Czechowska Y, Kinkingnehun S, Lemogne C, Le Bastard G, Lepage M, Garnero L, Colliot O, Fossati P 2009 Can voxel based morphometry, manual segmentation and automated segmentation equally detect hippocampal volume differences in acute depression? Neuroimage 45:29-37

46. Ashburner J 2009 Computational anatomy with the SPM software. Magn Reson Imaging 27:1163-1174 